\title{
DNA tetrahedron-mediated immune-sandwich assay for rapid and sensitive detection of PSA through a microfluidic electrochemical detection system
}

Dezhi Feng ${ }^{1,2,3}$, Jing Su${ }^{4}$, Yi Xu ${ }^{2}$, Guifang He ${ }^{2,5}$, Chenguang Wang ${ }^{2,3}$, Xiao Wang ${ }^{2,5}$, Tingrui Pan ${ }^{6}$, Xianting Ding ${ }^{4}$ and Xianqiang $\mathrm{Mi}^{1,2,3,7,8}$

\begin{abstract}
Prostate-specific antigen (PSA) is the most widely used biomarker for the early diagnosis of prostate cancer. Existing methods for PSA detection are burdened with some limitations and require improvement. Herein, we developed a novel microfluidic-electrochemical ( $\mu$ FEC) detection system for PSA detection. First, we constructed an electrochemical biosensor based on screen-printed electrodes (SPEs) with modification of gold nanoflowers (Au NFs) and DNA tetrahedron structural probes (TSPS), which showed great detection performance. Second, we fabricated microfluidic chips by DNA TSP-Au NF-modified SPEs and a PDMS layer with designed dense meandering microchannels. Finally, the $\mu \mathrm{FEC}$ detection system was achieved based on microfluidic chips integrated with the liquid automatic conveying unit and electrochemical detection platform. The $\mu \mathrm{FEC}$ system we developed acquired great detection performance for PSA detection in PBS solution. For PSA assays in spiked serum samples of the $\mu$ FEC system, we obtained a linear dynamic range of $1-100 \mathrm{ng} / \mathrm{mL}$ with a limit of detection of $0.2 \mathrm{ng} / \mathrm{mL}$ and a total reaction time $<25 \mathrm{~min}$. Real serum samples of prostate cancer patients presented a strong correlation between the "gold-standard" chemiluminescence assays and the $\mu \mathrm{FEC}$ system. In terms of operation procedure, cost, and reaction time, our method was superior to the current methods for PSA detection and shows great potential for practical clinical application in the future.
\end{abstract}

\section{Introduction}

Prostate cancer is one of the most common malignant tumors in the male population in the world. The latest data show that there were nearly 1.3 million new cases of prostate cancer and 359,000 prostate cancer-related deaths worldwide in 2018, being the fifth largest cause of death in men $^{1}$. PSA is secreted by prostate epithelial

\footnotetext{
Correspondence: Xianqiang Mi (mixq@mail.sim.ac.cn)

${ }^{1}$ Key Laboratory of Functional Materials for Informatics, Shanghai Institute of Microsystem and Information Technology, Chinese Academy of Sciences,

200050 Shanghai, China

${ }^{2}$ Shanghai Advanced Research Institute, Chinese Academy of Sciences, 201210 Shanghai, China

Full list of author information is available at the end of the article

These authors contributed equally: Dezhi Feng, Jing Su
}

cells and is a widely used biomarker for the early diagnosis of prostate cancer ${ }^{2}$. Immunoassay methods have been most widely used in the detection of PSA, such as enzyme-linked immunosorbent assay (ELISA) ${ }^{3,4}$, radioimmunoassay $(\mathrm{RIA})^{5}$, chemiluminescence immunoassay $(\text { CLIA })^{6}$, and fluorescent immunoassay (FIA $)^{7}$. However, these methods are burdened with limitations such as low sensitivity, complicated operations, and a lack of amenability with automatic detection. Thus, it is necessary to develop a rapid, sensitive, and automatic platform for PSA detection. An electrochemical biosensor is one such assay and is a simple, inexpensive, and accurate method for PSA measurement ${ }^{8-11}$.

\section{(c) The Author(s) 2021}

(c) (i) Open Access This article is licensed under a Creative Commons Attribution 4.0 International License, which permits use, sharing, adaptation, distribution and reproduction cc) in any medium or format, as long as you give appropriate credit to the original author(s) and the source, provide a link to the Creative Commons license, and indicate if changes were made. The images or other third party material in this article are included in the article's Creative Commons license, unless indicated otherwise in a credit line to the material. If material is not included in the article's Creative Commons license and your intended use is not permitted by statutory regulation or exceeds the permitted use, you will need to obtain permission directly from the copyright holder. To view a copy of this license, visit http://creativecommons.org/licenses/by/4.0/. 
One of the critical problems that should be solved for electrochemical detection is to improve its sensitivity. Many strategies have been used to improve the sensitivity of electrochemical biosensors, such as chemical derivatization, enzymatic reaction, and nanomaterial modification ${ }^{12-17}$. Among these strategies, nanomaterial modification has attracted increasing interest in recent years because it can improve the sensitivity of electrochemical biosensors by regulating the electrochemical interface through physical and chemical methods that change the structure and state of nanoparticles. One of the common strategies of nanomaterial modification is to deposit inorganic nanomaterials on electrodes. Pan et al. ${ }^{18}$ constructed a biosensor based on graphene oxide and dual-antibody-modified poly-L-lactide nanoparticles for PSA detection, achieving a detection limit of $1 \mathrm{ng} / \mathrm{mL}$. The detection limit of glycated hemoglobin A1c (HbA1c) achieved in our group was $5 \mu \mathrm{g} / \mathrm{mL}$ with the electrodeposition of gold nanoflowers (Au NFs) onto the electrode $^{19}$. Recently, DNA nanostructures have attracted much attention due to their high precision, structural stability, and ease of preparation ${ }^{20-23}$. In particular, threedimensional DNA tetrahedron structural probes (TSPs) were introduced to provide an ideal scaffold for the detection of $\mathrm{DNA}^{24}$, microRNA ${ }^{25}$, cocaine ${ }^{26}$, and $\mathrm{PSA}^{27}$. DNA TSPs could modulate the distribution and orientation of antibodies, which could decrease nonspecific adsorption on the electrode surface, control the nanospacing of immobilized antibodies, and then increase the detection sensitivity efficiently ${ }^{21,28-31}$. For example, Wen et al. ${ }^{26}$ utilized DNA TSPs to promote the efficient identification of aptamers with cocaine, reducing the detection limit to $33 \mathrm{nM}$ $(\sim 10 \mathrm{ng} / \mathrm{mL})$, which was three to four orders of magnitude higher than that of similar works; Chen et al. ${ }^{27}$ fabricated an ultrasensitive electrochemical immunosensor for PSA detection using DNA TSPs and acquired a lower detection limit of $1 \mathrm{pg} / \mathrm{mL}$, while the detection based on doublestranded DNA was $50 \mathrm{pg} / \mathrm{mL}$.

Another critical problem that should be solved for electrochemical detection is to achieve rapid and automatic detection. Microfluidic chips can integrate an entire laboratory into a single chip with compelling advantages of reduced sample volume, short reaction times, low cost, and portability. Microfluidics has become an important tool for the automatic detection of clinically relevant biomarkers. Electrochemical detectors could match microfluidic chips in size, cost, and portability, which showed great application prospects for point-of-care testing (POCT). Recently, great effort has been made to combine microfluidic devices with electrochemical biosensors $^{32-39}$. Oliveira et $\mathrm{al}^{32}$ constructed a disposable microfluidic immunoarray device (D $\mathrm{ID}$ ) based on a screen-printed array with eight electrodes for rapid detection of CA15-3; Yang et al. ${ }^{39}$ combined microfluidics with electrochemistry to achieve one-step detection of target molecules with an immune-sandwich reaction based on SPEs and obtained a detection limit of $500 \mathrm{pg} / \mathrm{mL}$ for PSA less than $30 \mathrm{~min}$.

In this work, we developed a novel microfluidicelectrochemical $(\mu \mathrm{FEC})$ detection system by introducing electrochemical biosensors, DNA TSPs, and microfluidic chips simultaneously for simple, rapid, and sensitive PSA detection. The electrochemical biosensor we constructed was based on a screen-printed electrode modified with gold nanoflowers (Au NFs) with the purpose of increasing the specific surface area and improving the chemical signal response. DNA TSPs were immobilized on the gold electrode surface by $\mathrm{Au}-\mathrm{S}$ bonds to provide a stable scaffold for the immune-sandwich analytical system. Biotin-anti-PSA (biotin-Ab1) and horseradish peroxidase-anti-PSA (HRPAb2) were attached to the DNA TSPs to form the classical "sandwich" structure. The PDMS microfluidic channels were tightly coupled on the screen-printed electrodes directly after plasma treatment. To obtain the optimal results, the $\mu \mathrm{FEC}$ system performance was investigated and optimized in this work, and the feasibility was demonstrated through the detection of PSA in real serum samples.

\section{Materials and methods \\ Reagents and apparatus}

The DNA sequences (A, B, C, D, and linker) shown in Supplementary Table S1 were synthesized by Sangon Biotech (Shanghai) Co., Ltd. Streptavidin (SA), 3,3'5,5'-tetramethylbenzidine (TMB) $/ \mathrm{H}_{2} \mathrm{O}_{2}$, and tris(2-carboxyethyl) phosphine (TCEP) were purchased from Sigma-Aldrich (St. Louis, MO, USA). PSA, biotinylated anti-PSA monoclonal antibody, and PSA antibody modified with horseradish peroxidase were purchased from Shanghai Linc-Bioscience Co. Ltd. Casein, bovine serum albumin (BSA), Tween 20, and other chemicals were purchased from Sinopharm Chemical Reagent Co. Ltd. All chemical reagents were prepared with ultrapure water from a Millipore Milli-Q water purification system $(18.2 \mathrm{M} \Omega \mathrm{cm}$ resistivity).

An HSBS16x electrochemical workstation and multiple channel screen-printed electrodes (SPEs) were purchased from HuasenXinke (Suzhou) Nanotechnology Co. Ltd. The silicon mold used in this work with special dense and meandering microfluidic channels was obtained from the Shanghai Institute of Applied Physics, Chinese Academy of Sciences. The DOWSIL ${ }^{\mathrm{TM}} 184$ polydimethylsiloxane (PDMS) prepolymer and curing agent were purchased from Dow Corning.

Healthy human blood samples were obtained from Shanghai Pudong New District Zhoupu Hospital and centrifuged at $3000 \mathrm{r} / \mathrm{m}$ for $10 \mathrm{~min}$ at $4{ }^{\circ} \mathrm{C}$, and the supernatant (serum) was collected and stored at $-80^{\circ} \mathrm{C}$. Prostate cancer patient serum samples were obtained from Shanghai Ninth People's Hospital, Shanghai 
JiaoTong University School of Medicine. The use of serum samples was approved by the Research Ethics Committees of hospitals. Serum samples used in this work were undiluted.

\section{Preparation of DNA TSPs}

Five single-stranded DNAs (A, B, C, D, and linker) were dissolved in TE buffer $(10 \mathrm{mM}$ Tris, $1 \mathrm{mM}$ EDTA, $\mathrm{pH}$ 8.0), yielding a final concentration of $100 \mu \mathrm{M}$. Then, $0.5 \mu \mathrm{L}$ of each strand was combined with $10 \mu \mathrm{L}$ TCEP $(30 \mathrm{mM})$ and $87.5 \mu \mathrm{L}$ TM buffer $(20 \mathrm{mM}$ Tris, $50 \mathrm{mM}$ $\mathrm{MgCl}_{2}, \mathrm{pH} \mathrm{8.0)}$, and the resulting mixture was heated to $95^{\circ} \mathrm{C}$ for $10 \mathrm{~min}$ and then cooled to $4{ }^{\circ} \mathrm{C}$ for $30 \mathrm{~s}$ using a $\mathrm{T} 100^{\mathrm{TM}}$ PCR Thermal Cycler. The final concentration of DNA TSPs was $0.5 \mu \mathrm{M}$.

\section{Preparation of polyacrylamide gel}

Six milliliters of polyacrylamide gel $(10 \%)$ was prepared with $2 \mathrm{~mL}$ of polyacrylamide (30\%), $1.2 \mathrm{~mL}$ of $5 \times \mathrm{TBE}$, $0.75 \mathrm{~mL}$ of $\mathrm{MgCl}_{2}$, and $2.05 \mathrm{~mL}$ of Milli-Q water, and the resulting mixture was mixed well. In total, $60 \mu \mathrm{L}$ of ammonium persulfate (APS) and $6 \mu \mathrm{L}$ of N,N,N,N'-tetramethylethylenediamine (TEMED) were added and mixed gently and quickly for further use.

\section{Construction of an electrochemical biosensor for PSA detection}

Each electrode was electrodeposited with $70 \mu \mathrm{L}$ of a $\mathrm{HAuCl}_{4}$ solution to obtain a layer of gold nanoflowers according to the reported protocol ${ }^{19}$. The parameters were as follows: deposition time, $150 \mathrm{~s}$; scan rate, $100 \mathrm{mV} / \mathrm{s}$; and deposition potential, $-200 \mathrm{mV}$. SPEs were placed in a wet box to avoid the electrode surface being dried during the reaction, and the working electrodes were incubated with $5 \mu \mathrm{L}$ of DNA TSPs $(0.5 \mu \mathrm{M})$ overnight at room temperature. Electrodes were rinsed with PBS ( $\mathrm{pH} 7.4)$. Each electrode was soaked in $50 \mu \mathrm{L}$ of blocking buffer $(1 \%$ casein and bovine serum albumin in PBS) at $37^{\circ} \mathrm{C}$ for $2 \mathrm{~h}$ to eliminate nonspecific binding sites. Five microliters of SA $(200 \mu \mathrm{g} / \mathrm{mL})$ were linked with biotinylated DNA TSPs (Linker) at $37^{\circ} \mathrm{C}$ for $30 \mathrm{~min}$. After being washed with PBST, biotin-Ab1 $(100 \mu \mathrm{g} / \mathrm{mL})$ was linked with SA by dropping $5 \mu \mathrm{L}$ of biotin- $\mathrm{Ab} 1$ at $37^{\circ} \mathrm{C}$ for $1 \mathrm{~h}$ to form biotin-Ab1/SA/DNA TSPs/Au NFs/SPEs, and the excess reagents were washed with PBS. The resulting biosensor was stored at $4{ }^{\circ} \mathrm{C}$ for further use.

The concentrations of PSA were diluted with PBS from the initial concentration $(0.5 \mathrm{mg} / \mathrm{mL})$ to concentrations ranging from 0 to $300 \mathrm{ng} / \mathrm{mL}$, and an HSBS16x electrochemical workstation was used to perform measurements by cyclic voltammetry (CV) and amperometry (IT). First, $5 \mu \mathrm{L}$ PSA was coated on the working electrodes at $37^{\circ} \mathrm{C}$ for $1 \mathrm{~h}$. Then, $5 \mu \mathrm{L}$ of $10 \mu \mathrm{g} / \mathrm{mL}$ HRP-Ab2 was added to the electrode surface at $37^{\circ} \mathrm{C}$ for $1 \mathrm{~h}$, and redundant reagents were washed away with PBS and dried under $\mathrm{N}_{2}$. Finally, each electrode was rinsed with PBST as above and soaked with $50 \mu \mathrm{L}$ of TMB $\left(\mathrm{H}_{2} \mathrm{O}_{2}\right)$ solution, and reactions on SPEs were detected by an electrochemical workstation. $\mathrm{CV}$ experiments were conducted at a scanning rate of $0.1 \mathrm{~V} / \mathrm{s}$ ranging from $-0.3 \mathrm{~V}$ to $0.7 \mathrm{~V}$. The parameters of IT were as follows: initial potential, $-0.1 \mathrm{~V}$; run time, $300 \mathrm{~s}$; sample interval, $0.5 \mathrm{~s}$; current sensitivity, $1 \times \mathrm{e}^{-5} \mathrm{~A} / \mathrm{V}$; and rest time, $3 \mathrm{~s}$.

\section{Development of the $\mu \mathrm{FEC}$ system for PSA detection}

The mixtures of DOWSIL ${ }^{\text {TM }} 184$ prepolymer solution and curing agent (10:1) were poured onto a silicon wafer, cured at $80^{\circ} \mathrm{C}$ for $1 \mathrm{~h}$, and peeled off. A shaped polydimethylsilicone (PDMS) layer was cut into individual chips, and inlet and outlet holes were punched for use. The PDMS layer was $40 \mathrm{~mm}$ in length and $20 \mathrm{~mm}$ in width, and the channels in the PDMS layer were $400 \mu \mathrm{m}$ in width and $115 \mu \mathrm{m}$ in height. Each electrode $(10 \mathrm{~mm}$ width) of the SPEs used in this work comprised a carbon working electrode (3 $\mathrm{mm}$ diameter), a carbon counter electrode, and an Ag reference electrode (Supplementary Fig. S1). The SPEs were pretreated with biotin-Ab1/SA/DNA TSPs/Au NFs to construct an electrochemical biosensor. The modified SPEs and PDMS layer were bonded together by a YZD08-2- $\mathrm{CO}_{2}$ plasma cleaner for $5 \mathrm{~s}$ (the power of the plasma cleaner was $200 \mathrm{~W}$ ) under the protection of Eppendorf (EP) tube caps on the SPE surface. A syringe pump was used to inject PBS washing solution and other reagents into the chip through transparent microtubing, and an electrochemical workstation was used for measurement.

For PSA detection in PBS solution or spiked serum samples, the concentrations of PSA in PBS solution or healthy human serum solution were obtained by gradient dilution with concentrations ranging from 0 to $100 \mathrm{ng} / \mathrm{mL}$. Ten microliters of PBS solution or healthy human serum solution were injected into the chip at a flow rate of $8 \mu \mathrm{L} / \mathrm{min}$. With the channel and electrode surfaces washed with PBS, $10 \mu \mathrm{L}$ HRP-Ab2 was added to the surface of electrodes at the same flow rate. After being washed again, $40 \mu \mathrm{L}$ of TMB $\left(\mathrm{H}_{2} \mathrm{O}_{2}\right)$ solution was injected into the chip, and PSA was detected by an electrochemical workstation. For PSA detection in real serum samples, the detection protocol was the same as in PBS solution and spiked serum samples.

\section{Results and discussion}

Design strategy of the electrochemical biosensor and $\mu \mathrm{FEC}$ detection system

As shown in Scheme 1a, we first immobilized DNA TSPs onto the $\mathrm{Au} \mathrm{NF}$ electrode surface through $\mathrm{Au}-\mathrm{S}$ bonds ${ }^{40}$. Each DNA TSP consists of five single-stranded DNAs (A, B, $\mathrm{C}, \mathrm{D}$, and linker) in this work, which have a stable and rigid structure. Then, we utilized the remarkably high affinity 

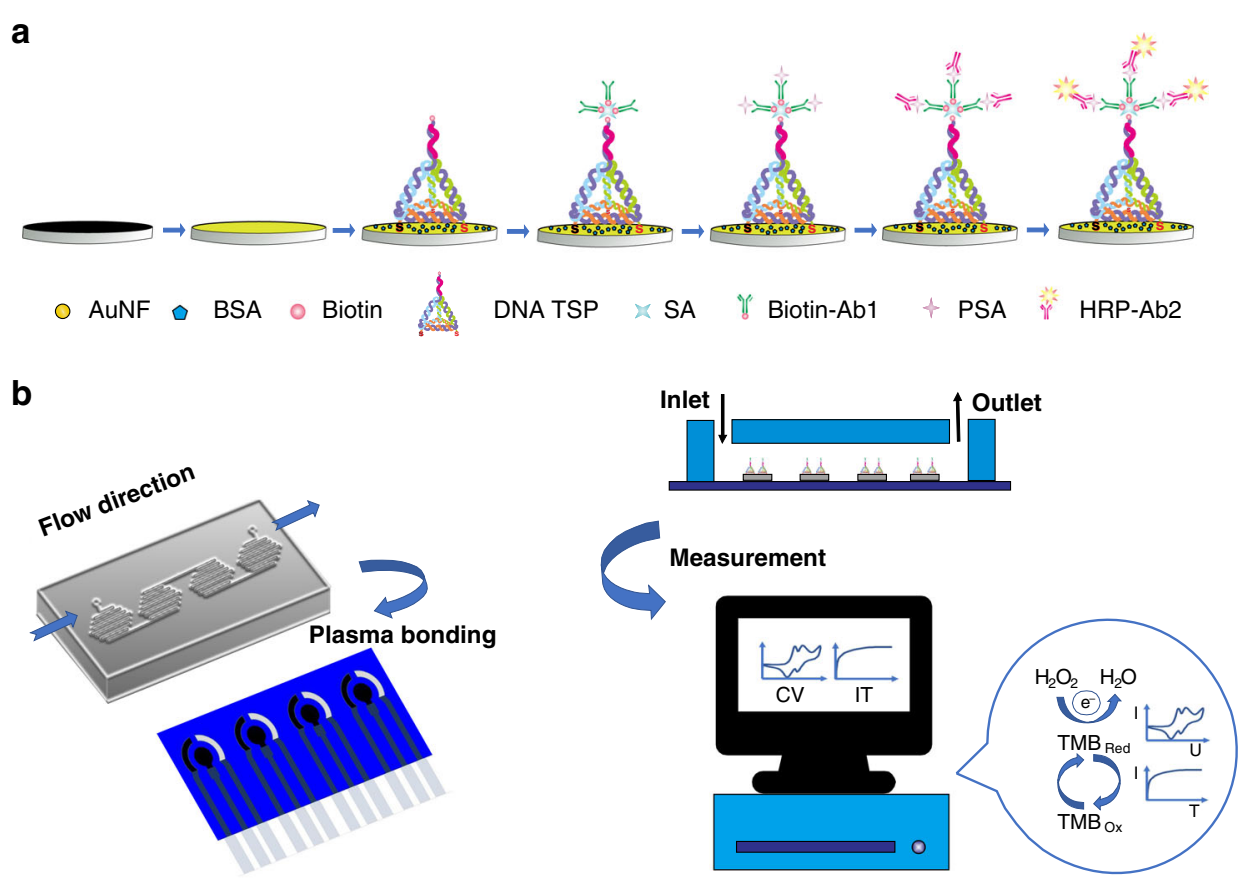

Scheme 1 Schematic illustration of the $\boldsymbol{\mu F E C ~ s y s t e m . ~ a ~ S c h e m e ~ o f ~ t h e ~ c o n s t r u c t i o n ~ o f ~ t h e ~ e l e c t r o c h e m i c a l ~ b i o s e n s o r . ~} \mathbf{b}$ Scheme of the development of the $\mu$ FEC system for PSA detection

between SA and biotin to enhance the detection sensitivity. Biotin-Ab1 was connected to the DNA TSPs through the biotin-SA interaction. The classical antibody-antigenantibody sandwich structure was fabricated by connecting PSA and HRP-Ab2. Finally, we constructed an electrochemical biosensor with a carbon interface immunesandwich structure mediated with DNA TSPs and obtained electrochemical signals by $\mathrm{CV}$ and IT, in which $\mathrm{HRP}$ catalyzes the reduction of $\mathrm{H}_{2} \mathrm{O}_{2}$ and generates signals in the presence of TMB $\left(\mathrm{H}_{2} \mathrm{O}_{2}\right)$.

To achieve rapid and automatic PSA detection, we employed microfluidic chips to develop a $\mu$ FEC detection system (Scheme 1b) and adopted dense meandering microchannels $^{39,41}$ for sequential and stable delivery of reagents over the SPE surface. We designed a fourelectrode SPE array that was bonded with the PDMS layer (Supplementary Fig. S1) by $\mathrm{O}_{2}$ plasma under the protection of EP tube caps on the SPE surface to avoid plasma irradiation-induced damage to antibodies. Reagents were injected into the chip with the settled volume and flow rate by the $\mu \mathrm{FEC}$ detection system. The $\mu \mathrm{FEC}$ detection system connected to the unit of liquid automatic conveying and electrochemical detection platform is shown in Supplementary Fig. S2.

\section{Characterization of the Au NFs-SPE and DNA TSPs}

Scanning electron microscopy (SEM) was employed to characterize the surface of the SPEs. Figure $1 \mathrm{a}, \mathrm{b}$ shows the SEM results of the bare carbon electrodes and gold nanoflower-modified electrodes. It was obvious that the carbon electrode had a relatively smooth surface, while the Au NF-modified electrodes had a highly rough surface area. After detailed analysis, we found that Au NFs were widely distributed on the electrode surface and that the diameter of the particles was approximately $78.831 \mathrm{~nm}$, ranging from 7 to $237 \mathrm{~nm}$. Electrodes modified with $\mathrm{Au}$ NFs could increase the specific surface area and improve the performance of electron transfer and chemical signal response.

We selected two single-stranded DNAs (A, D), two double-stranded DNAs (AB, CD), two triple-stranded DNAs (ABC, BCD), one four-stranded DNA (ABCD), and one five-stranded DNA (ABCDL) to assemble tetrahedra, and the formation of the tetrahedron in solution was confirmed through polyacrylamide gel electrophoresis (PAGE). As shown in Fig. 1c, negatively charged DNA molecules move toward the anode in the electric field, and the molecular weights of eight lanes are in descending order, which is close to the theoretical value. DNA TSPs shifted slower than the combinations of triple-strand, double-strand and single-strand DNA, which confirmed that the successful formation of the DNA TSPs as the size and configuration of the DNA molecule influenced the migration speed. Dynamic light scattering (DLS) and atomic force microscopy (AFM) were then performed to characterize the DNA TSPs. Each tetrahedron is 


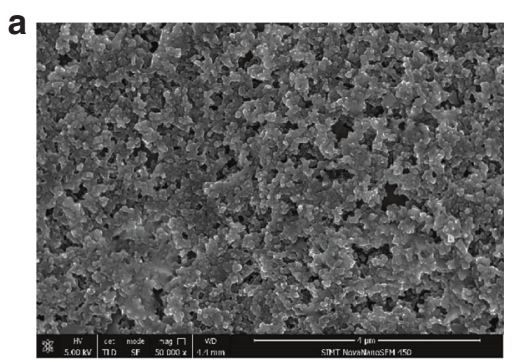

C

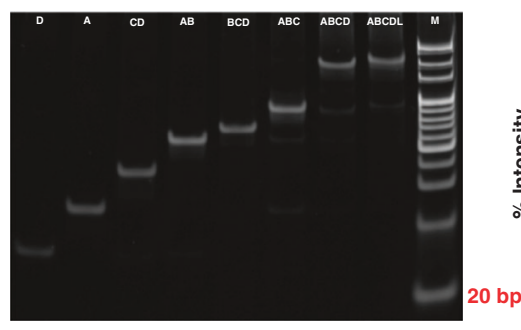

d

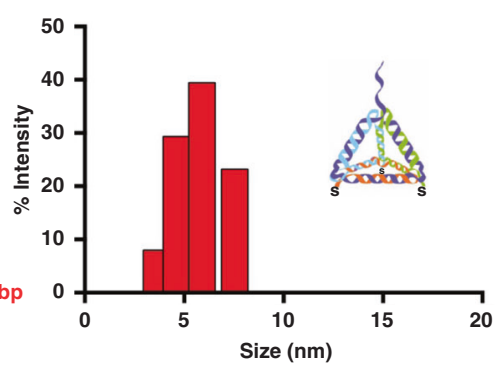

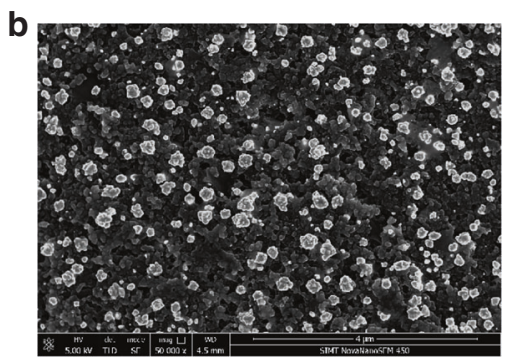

e

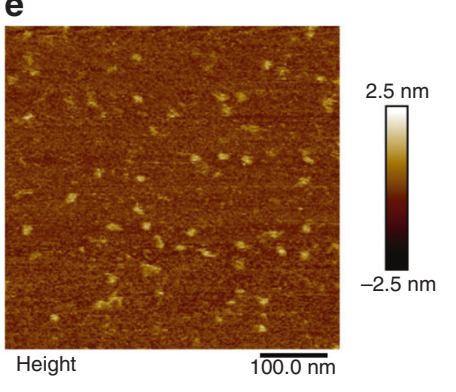

Fig. 1 Characterization of the Au NFs-SPE and DNA TSPs. a, b SEM results of the bare carbon electrode and the electrodes after Au NF deposition. The scale value was $4 \mu \mathrm{m}$. c Gel electrophoresis image of different DNA strands. d DLS results of DNA TSPs. e AFM results of DNA TSPS

assembled from three 55-base, one 80-base, and one 15base oligonucleotide, which form the structure with six 17-base pair edges (each base pair was approximately $0.34 \mathrm{~nm})$ and one double-strand branch $^{42}$. As shown in Fig. 1d, the size of the nanostructure acquired from DLS was approximately $5.823 \mathrm{~nm}$, which was close to the calculated value $(5.78 \mathrm{~nm})$. The triangle in the AFM image (Fig. 1e) shows that the tetrahedral structure was formed successfully. All these results showed the correct formation of DNA TSPs.

\section{Optimization of the experimental conditions of the $\mu \mathrm{FEC}$ detection system}

Some experimental conditions influencing the performance of the $\mu \mathrm{FEC}$ detection system were optimized. The optimization of these conditions was indicated by the signal-to-noise ratio $\left(\mathrm{SNR}=\right.$ Current $_{\mathrm{PSA}-10 \mathrm{ng} / \mathrm{mL}} /$ Curren$t_{\text {blank }}$ ) at a concentration of $10 \mathrm{ng} / \mathrm{mL}$ PSA, which was close to the value of clinical diagnostic criteria for prostate cancer $^{8,43}$.

DNA TSP played a key role in providing the scaffold of the sandwich structure, and HRP-Ab2 benefited the formation of sandwich structure and signal catalysis, which directly affected the analytical performance of immunoassays. As shown in Fig. 2a, the SNR reached a maximum at a concentration of $0.5 \mu \mathrm{M}$ and was higher than that at $0.25 \mu \mathrm{M}$ or $1 \mu \mathrm{M}$, suggesting that the optimal concentration of DNA TSPs was $0.5 \mu \mathrm{M}$. In Fig. 2b, the SNR increased significantly when the HRP-Ab2 concentration increased. However, when the concentration of
HRP-Ab2 was higher than $10 \mu \mathrm{g} / \mathrm{mL}$, the SNR decreased slightly, and $10 \mu \mathrm{g} / \mathrm{mL}$ was chosen as the optimal concentration of HRP-Ab2. The affinity between biotin and SA was also critical, which influenced the stability of the sandwich structure. A sufficient and appropriate concentration of biotin-Ab1 could ensure that PSA was captured effectively on the electrode surface. Here, the concentration of SA and biotin-Ab1 was optimized. Figure 2c shows that the SNR increased gradually with increasing SA concentration and reached a maximum at $200 \mu \mathrm{g} / \mathrm{mL}$, which was higher than that of other concentrations. Similarly, in Fig. 2d, we found that the SNR increased to the maximum at $100 \mu \mathrm{g} / \mathrm{mL}$ biotin-Ab1 and was higher than that at 25, 50, or $200 \mu \mathrm{g} / \mathrm{mL}$. Excess SA and biotin-Ab1 lowered the binding efficiency conversely, and we selected $200 \mu \mathrm{g} / \mathrm{mL}$ and $100 \mu \mathrm{g} / \mathrm{mL}$ as the optimal concentrations of SA and biotin-Ab1, respectively, to ensure that the reaction was completely finished.

In addition to the above four conditions, the volume and flow rate of reagents would also impact the $\mu \mathrm{FEC}$ detection system, which influenced the binding efficiency of antigen-antibody and the stability of the sandwich structure in the system. Based on the optimized concentrations of DNA TSP, HRP-Ab2, SA, and biotin-Ab1, the optimization of these two conditions was subsequently indicated by the current at a PSA concentration of $100 \mathrm{ng} / \mathrm{mL}$. Figure $2 \mathrm{e}$ shows that a $10 \mu \mathrm{L}$ sample volume produced a maximal signal for PSA detection compared to other sample volumes. Then, $10 \mu \mathrm{L}$ was selected as the optimal volume for 

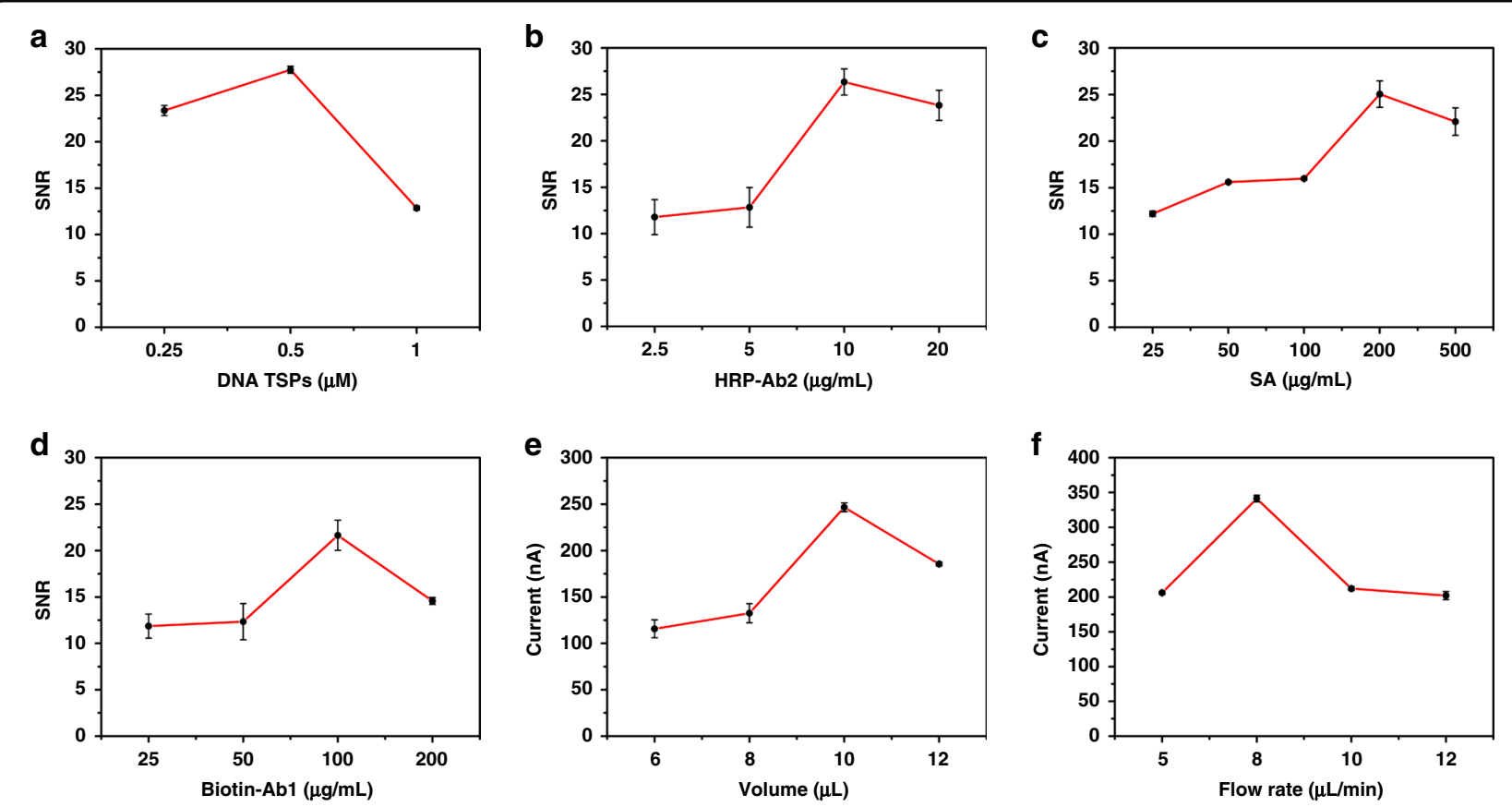

Fig. 2 Optimization of the experimental conditions of the $\mu \mathrm{FEC}$ detection system. Optimization of the concentrations of (a) DNA TSPS and (b) HRP-Ab2 (c) SA (d) biotin-Ab1 and the conditions of (e) volume and (f) flow rate of $\mu$ FEC detection system. The error bars show the standard deviation of four replicate determinations

further use. Similarly, we found that a flow rate of $8 \mu \mathrm{L} /$ min led to sufficiently strong signals within a reasonable time (Fig. 2f), while other flow rates $(5,10$, and $12 \mu \mathrm{L} /$ min) could cause an inadequate response with a lower detection current, suggesting that $8 \mu \mathrm{L} / \mathrm{min}$ was the optimal flow rate of the system.

\section{Analytical performance of the $\mu \mathrm{FEC}$ detection system}

Under the optimized conditions determined above, we performed PSA detection in PBS by the electrochemical biosensor. First, the signal increased gradually with increasing PSA concentration and remained stable when the concentration was higher than $100 \mathrm{ng} / \mathrm{mL}$ (Fig. 3a). Then, a linear relationship between the current and the PSA concentration from 0 to $100 \mathrm{ng} / \mathrm{mL}$ was acquired (Fig. 3b). The linear regression equation was expressed as current $=110.274 *[\mathrm{PSA}]+74.134$ with a correlation coefficient of 0.972 . As the signal at a concentration of $2 \mathrm{pg} / \mathrm{mL}$ was obviously higher than the threshold, which was equal to the blank control signal plus three standard deviations (3 SD), a limit of detection (LOD) of $2 \mathrm{pg} / \mathrm{mL}$ could be obtained (Fig. 3b, inset). The high detection sensitivity benefited greatly from the introduction of $\mathrm{Au}$ NFs, DNA TSPs, and immune-sandwich structures, which were three orders of magnitude higher than $4 \mathrm{ng} / \mathrm{mL}$, indicating a risk of prostate cancer according to the previous works ${ }^{8,43}$.
The specificity test for PSA $(10 \mathrm{ng} / \mathrm{mL})$ was also carried out against alpha-fetoprotein (AFP, $100 \mathrm{ng} / \mathrm{mL}$ ) and carcinoembryonic antigen $(\mathrm{CEA}, 100 \mathrm{ng} / \mathrm{mL})$. As shown in Fig. 3c, it was obvious that the signal was as low as that of the blank compared to the higher concentrations of AFP and CEA, but it exhibited a strong signal response when a lower concentration of PSA was detected. In the presence of PSA, the current of the biosensor increased significantly, which benefited from the stable electrochemical signal generated by the classical sandwich structure of antibody-antigen-antibody. The results showed that the biosensor had a better discrimination ability for PSA than AFP and CEA.

To further realize rapid and automatic detection, we performed PSA assays using the $\mu$ FEC system. In Fig. $3 d$, the results indicated that the regression equation was current $=3.517^{*}[\mathrm{PSA}]+192.228$ with a correlation coefficient of 0.989. The calibration curve of PSA in PBS solution was in the concentration range of $1-100 \mathrm{ng} / \mathrm{mL}$ with an LOD of $0.2 \mathrm{ng} / \mathrm{mL}$ (Fig. 3d, inset). The reason for the lower sensitivity of the $\mu \mathrm{FEC}$ system was that we sacrificed the longer incubation time and the larger reagent volume in exchange for a rapid test with less manual operation within $25 \mathrm{~min}$.

We also compared the performance of the $\mu \mathrm{FEC}$ system with some microfluidic immunoassay methods for PSA detection (Table 1). Our $\mu$ FEC system showed an obviously 

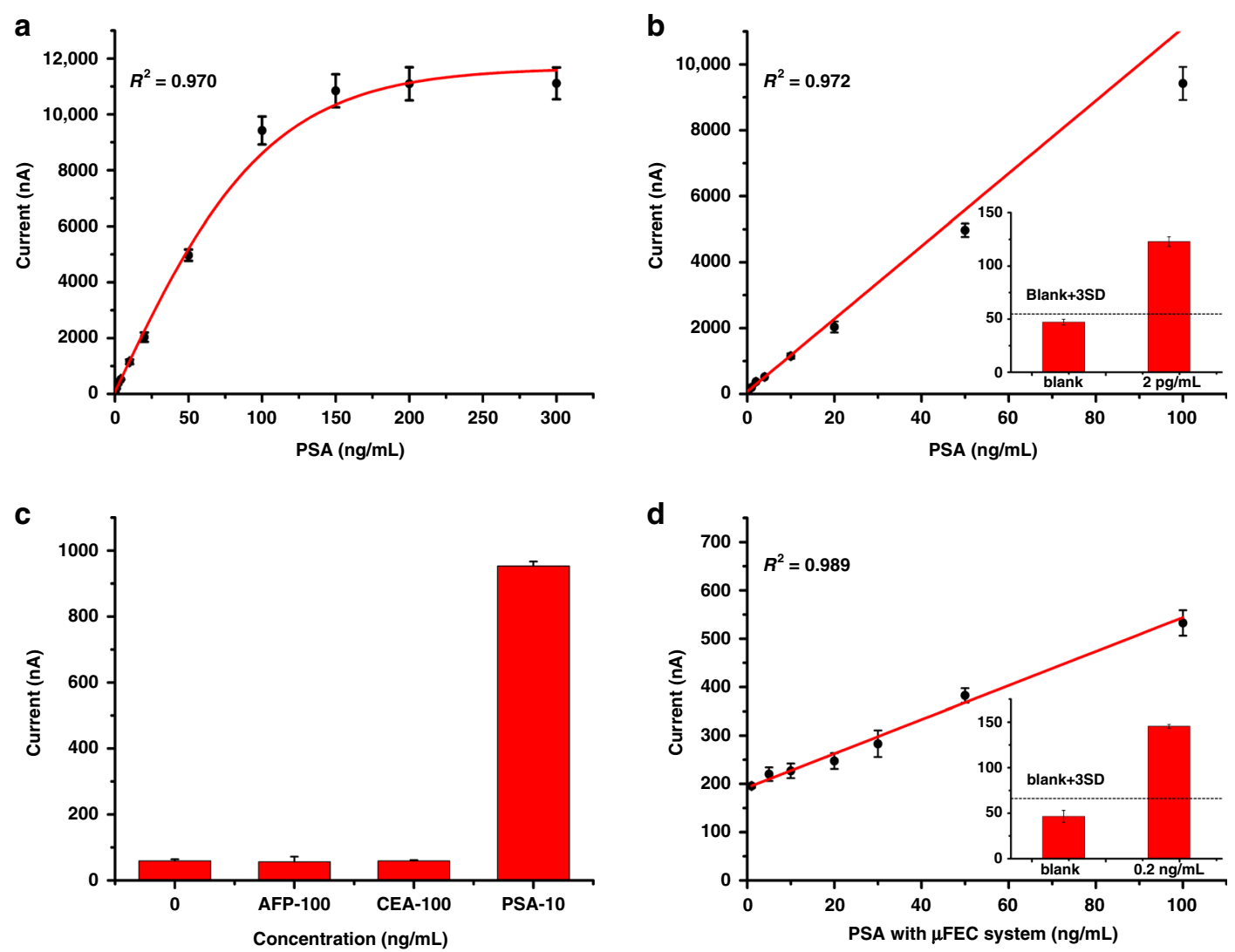

Fig. 3 Analytical performance of the $\mu$ FEC detection system. a The nonlinear calibration curve for PSA detection in PBS with concentrations of 0, $0.002,0.003,1,2,4,10,20,50,100,150,200$, and $300 \mathrm{ng} / \mathrm{mL}$ by the electrochemical biosensor. $\mathbf{b}$ Linear calibration curve for PSA detection in PBS at concentrations of $0,0.002,0.003,1,2,4,10,20,50$, and $100 \mathrm{ng} / \mathrm{mL}$ by the electrochemical biosensor. Inset: Histogram showing the LOD of PSA detection by the electrochemical biosensor, and the dashed lines represent the threshold (blank + 3 SD). c Specificity study of the electrochemical biosensor. $\mathbf{d}$ The linear calibration curve for PSA detection in PBS at concentrations of 1, 5, 10, 20, 30, 50, and $100 \mathrm{ng} / \mathrm{mL}$ with a $\mu \mathrm{FEC}$ detection system. Inset: Histogram showing the LOD of PSA detection with the $\mu$ FEC detection system, and the dashed lines represent the threshold (blank + 3SD). The error bars show the standard deviation of four replicate determinations

Table 1 Comparison of the $\mu$ FEC detection system with other methods for PSA detection

\begin{tabular}{|c|c|c|c|c|}
\hline Method & Dynamic range (ng/mL) & LOD (ng/mL) & Reaction time (min) & Reference \\
\hline An intelligent microscale electrochemical device & $0.5-100$ & 0.5 & 30 & 39 \\
\hline Microfluidic chip-enzyme immunoassay & $3.2-50$ & 3.2 & 30 & 44 \\
\hline A microfluidic immunoreaction platform & $0.1-20$ & 0.1 & 40 & 45 \\
\hline Microfluidic paper-based analytical device & $1-50$ & 0.3 & 45 & 46 \\
\hline Microfluidic paper-based fluorometric immunodevice & $1-40$ & 0.4 & 45 & 47 \\
\hline$\mu F E C$ detection system in this work & $1-100$ & 0.2 & 25 & \\
\hline
\end{tabular}

lower limit of detection than that of the chip-enzyme immunoassay platform ${ }^{44}$. The high sensitivity we achieved was attributed to the use of SPEs modified with Au NFs and DNA TSPs. Compared with other microfluidic immunoassay methods with similar sensitivity ${ }^{39,45-47}$, our system had a wider linear range, which allowed the detection of complicated and high concentrations of samples. In comparison to other methods in PSA detection ${ }^{39,44-47}$, our method integrated liquid conveying, immunoreaction, and detection in one system and accomplished all steps in less than $25 \mathrm{~min}$. Based on these advantages, our $\mu \mathrm{FEC}$ system meets the requirement of clinical POCT. 

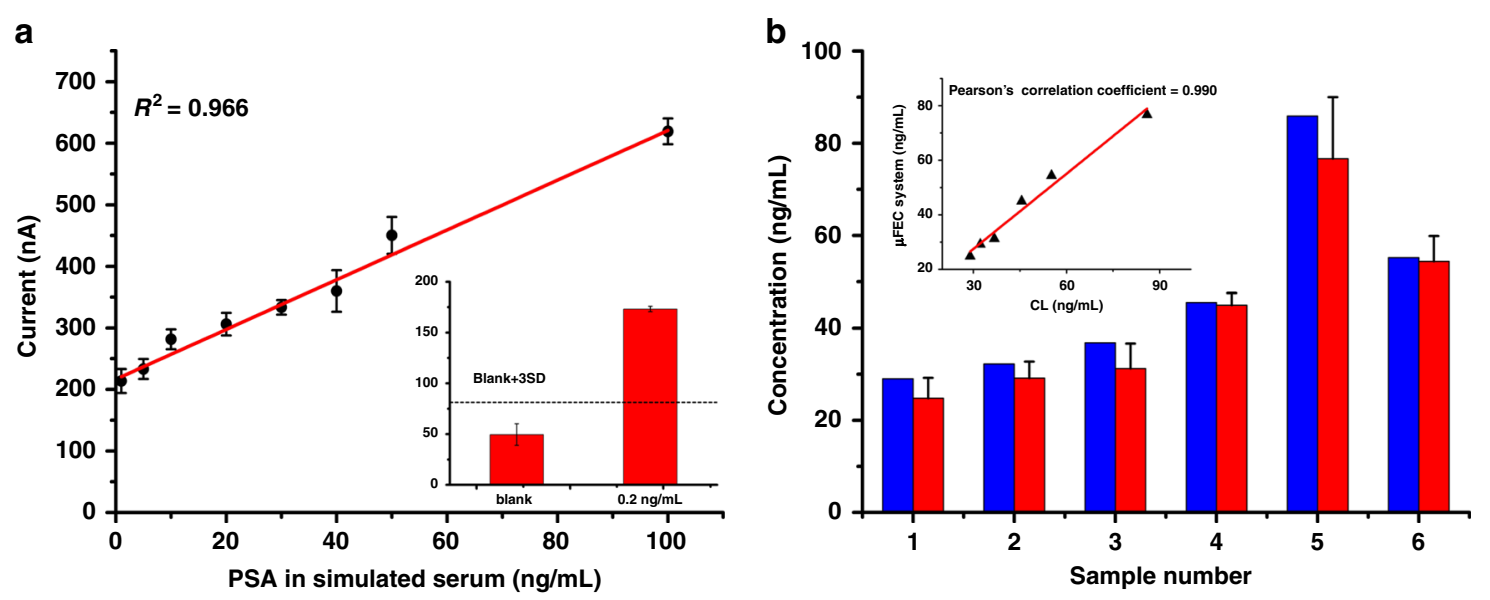

Fig. 4 Application to complex sample analysis of the $\mu \mathrm{FEC}$ detection system. a The linear calibration curve of PSA detection in simulated serum with concentrations of $1,5,10,20,30,40,50$, and $100 \mathrm{ng} / \mathrm{mL}$ by the $\mu \mathrm{FEC}$ detection system. Inset: Histogram showing the LOD of PSA detection in simulated serum, and the dashed lines represent the threshold (blank + 3 SD). b Comparisons between the results by CL from the hospital and our $\mu \mathrm{FEC}$ detection system. Blue columns represent the reference value by the $\mathrm{CL}$, and red columns represent the detection value of the $\mu \mathrm{FEC}$ detection system. The error bars show the standard deviation of four replicate determinations. Inset: Calibration curve of correlation analysis between two methods

\section{Application to spiked serum and real serum sample analysis of the $\mu \mathrm{FEC}$ detection system}

To investigate the clinical practical application of the $\mu$ FEC detection system, we continued to detect PSA in spiked serum samples with different PSA concentrations, which were obtained by gradient dilution. As shown in Fig. 4a, the current increased gradually with increasing PSA concentration, and a linear relationship between the current and PSA concentrations from $1 \mathrm{ng} / \mathrm{mL}$ to $100 \mathrm{ng} /$ $\mathrm{mL}$ was acquired. The linear equation was current $=$ $4.042 *[\mathrm{PSA}]+216.62$ with a correlation coefficient of 0.966 , and the limit of detection (LOD) was $0.2 \mathrm{ng} / \mathrm{mL}$ (Fig. 4a, inset). Previous reports have indicated that a PSA level of $4 \mathrm{ng} / \mathrm{mL}$ is usually applied to indicate a risk of prostate cancer $^{8,43}$. A PSA concentration of $0.2 \mathrm{ng} / \mathrm{mL}$ is enough to monitor the recurrence of prostate cancer.

Then, we applied our $\mu$ FEC detection system to detect PSA in recent real serum samples after centrifugal treatment of six prostate cancer patients with different stages. The detection results of the chemiluminescence (CL) method from the hospital and the $\mu$ FEC system are shown in Supplementary Table S2. As shown in Fig. 4b, the two methods exhibited a strong correlation, with a Pearson's correlation coefficient of 0.990 (Fig. 4b, inset). As CL is the "gold-standard" assay in hospitals for PSA detection, it was well manifested that our $\mu$ FEC detection system has complicated sample analysis capability and could be used in clinical sample analysis procedures. The total reaction time was less than $25 \mathrm{~min}$, which means it is useful for POCT and has potential clinical applications for routine screening of prostate cancer.

\section{Conclusions}

In summary, we developed a novel microfluidicelectrochemical $(\mu \mathrm{FEC})$ system for the detection of PSA. The electrochemical biosensor was first constructed by SPEs modified with Au NFs and DNA TSPs, which achieved a linear dynamic range of $0-100 \mathrm{ng} / \mathrm{mL}$ and an LOD of $2 \mathrm{pg} / \mathrm{mL}$. Then, we developed a microfluidic-electrochemical detection system for rapid and automatic PSA detection based on microfluidic chips together with an electrochemical biosensor. Under optimized conditions, we obtained a good linear range $(1-100 \mathrm{ng} / \mathrm{mL})$ and detection limit $(0.2 \mathrm{ng} / \mathrm{mL})$ with a total reaction time of less than $25 \mathrm{~min}$. Moreover, we successfully detected PSA in real clinical samples. This system we developed had the following features: (i) DNA TSPs were used as the basis to form and stabilize the sandwich structure and were in favor of decreasing the nonspecific adsorption on the SPE surface and improving the binding efficiency; (ii) classical immune-sandwich structures benefit the stable, reliable and sensitive detection of PSA; (iii) this technique was easy to use without professional skills and complicated instruments; and (iv) this method has the potential to realize the parallel detection of multiple biomarkers. On the basis of this research, we believe this system will hopefully be used for POCT and have a brilliant future for practical clinical applications.

\footnotetext{
Acknowledgements

We are thankful for support from the Program of Shanghai Academic/

Technology Research Leader (20XD1404600), Shanghai Municipal Science and Technology Commission (Grant nos. 20511107600, 19511107100, 19511107102), Chinese Academy of Sciences (KFJ-STS-QYZD-2021-08-002),
} 
National Key Research and Development Program of China (Grant no. 2016YFC0100600), Shenzhen Fundamental Research Program (JCYJ20170413164102261), and Guangdong Program (2016ZT06D631).

\section{Author details}

'Key Laboratory of Functional Materials for Informatics, Shanghai Institute of Microsystem and Information Technology, Chinese Academy of Sciences, 200050 Shanghai, China. ${ }^{2}$ Shanghai Advanced Research Institute, Chinese Academy of Sciences, 201210 Shanghai, China. ${ }^{3}$ University of Chinese Academy of Sciences, 100049 Beijing, China. ${ }^{4}$ State Key Laboratory of Oncogenes and Related Genes, Institute for Personalized Medicine, School of Biomedical Engineering, Shanghai Jiao Tong University, 200030 Shanghai, China. ${ }^{5}$ School of Life Sciences, Shanghai University, 200444 Shanghai, China. ${ }^{6}$ Shenzhen Institutes of Advanced Technology, Chinese Academy of Science, 518055 Shenzhen, China. ${ }^{7}$ CAS Center for Excellence in Superconducting Electronics, (CENSE), 200050 Shanghai, China. ${ }^{8}$ Key Laboratory of Systems Health Science of Zhejiang Province, Hangzhou Institute for Advanced Study, University of Chinese Academy of Sciences, Chinese Academy of Sciences, 310024 Hangzhou, China

\section{Author contributions}

D.F. and J.S. contributed equally to this work. X.M. conceived and directed the study; J.S., D.F., and X.M. designed and performed the experiments and wrote the paper; Y.X., G.H., C.W., X.W., T.P., and X.D. helped with data analysis and investigation. All authors approved the final paper.

\section{Conflict of interest}

The authors declare no competing interests.

Supplementary information The online version contains supplementary material available at https://doi.org/10.1038/s41378-021-00258-x.

Received: 12 November 2020 Revised: 25 February 2021 Accepted: 8 March 2021

Published online: 25 April 2021

\section{References}

1. Bray, F. et al. Global cancer statistics 2018: GLOBOCAN estimates of incidence and mortality worldwide for 36 cancers in 185 countries. CA: A Cancer J. Clin. 68, 394-424 (2018)

2. Karim-Kos, H. E. et al. Recent trends of cancer in Europe: a combined approach of incidence, survival and mortality for 17 cancer sites since the 1990s. Eur. J. Cancer 44, 1345-1389 (2008).

3. Becker, C. \& Lilja, H. Individual prostate-specific antigen (PSA) forms as prostate tumor markers. Clin. Chim. Acta 257, 117-132 (1997).

4. Fenner, A. Novel "inverse sensitivity" enzyme-linked crystal-growth assay to detect ultralow PSA levels. Nat. Rev. Urol. 9, 354-354 (2012).

5. Graves, H. C., Wehner, N. \& Stamey, T. A. Ultrasensitive radioimmunoassay of prostate-specific antigen. Clin. Chem. 38, 735 LP-735742 (1992).

6. Fu, Z., Yan, F., Liu, H., Yang, Z. \& Ju, H. Channel-resolved multianalyte immunosensing system for flow-through chemiluminescent detection of a-fetoprotein and carcinoembryonic antigen. Biosens. Bioelectron. 23, 1063-1069 (2008).

7. Mackness, B. C., Chourb, S., Farris, L. R. \& McDonald, M. J. Polymer-proteinenhanced fluoroimmunoassay for prostate-specific antigen. Anal. Bioanal. Chem. 396, 681-686 (2010).

8. Healy, D. A., Hayes, C. J., Leonard, P., McKenna, L. \& O'Kennedy, R. Biosensor developments: application to prostate-specific antigen detection. Trends Biotechnol. 25, 125-131 (2007).

9. Lin, Y.-Y. et al. A nanoparticle label/immunochromatographic electrochemical biosensor for rapid and sensitive detection of prostate-specific antigen. Biosens. Bioelectron. 23, 1659-1665 (2008).

10. Wei, Y. et al. Dual-responsive electrochemical immunosensor for prostate specific antigen detection based on Au-CoS/graphene and $\mathrm{CeO} /$ /ionic liquids doped with carboxymethyl chitosan complex. Biosens. Bioelectron. 94, 141-147 (2017).
11. Yu, Q. et al. Novel electrochemical biosensor based on cationic peptide modified hemin/G-quadruples enhanced peroxidase-like activity. Biosens. Bioelectron. 107, 178-183 (2018).

12. Garcia, P. T., Guimarães, L. N., Dias, A. A., Ulhoa, C. J. \& Coltro, W. K. T. Amperometric detection of salivary a-amylase on screen-printed carbon electrodes as a simple and inexpensive alternative for point-of-care testing. Sens. Actuators B: Chem. 258, 342-348 (2018).

13. Okuno, J. et al. Label-free immunosensor for prostate-specific antigen based on single-walled carbon nanotube array-modified microelectrodes. Biosens. Bioelectron. 22, 2377-2381 (2007).

14. Wei, J. et al. Dual-modal split-type immunosensor for sensitive detection of microcystin-LR: enzyme-induced photoelectrochemistry and colorimetry. Anal. Chem. 90, 9606-9613 (2018).

15. Yuan, Z. et al. A trimetallic CuAuPd nanowire as a multifunctional nanocomposites applied to ultrasensitive electrochemical detection of Sema3E. Biosens. Bioelectron. 145, 111677 (2019).

16. Lu, N. et al. The application of silicon nanowire field-effect transistor-based biosensors in molecular diagnosis. Chin. Sci. Bull. 61, 442-452 (2016).

17. Liu, Y. \& Yobas, L. Label-free specific detection of femtomolar cardiac troponin using an integrated nanoslit array fluidic diode. Nano Lett. 14, 6983-6990 (2014).

18. Pan, L.-H. et al. An electrochemical biosensor to simultaneously detect VEGF and PSA for early prostate cancer diagnosis based on graphene oxide/ssDNA PLLA nanoparticles. Biosens. Bioelectron. 89, 598-605 (2017).

19. Wang, X. et al. Gold nano-flowers (Au NFs) modified screen-printed carbon electrode electrochemical biosensor for label-free and quantitative detection of glycated hemoglobin. Talanta 201, 119-125 (2019).

20. Lu, N. et al. Charge transport within a three-dimensional DNA nanostructure framework. J. Am. Chem. Soc. 134, 13148-13151 (2012).

21. Pei, $\mathrm{H}$. et al. A DNA nanostructure-based biomolecular probe carrier platform for electrochemical biosensing. Adv. Mater. 22, 4754-4758 (2010).

22. Pei, H., Zuo, X., Zhu, D., Huang, Q. \& Fan, C. Functional DNA nanostructures for theranostic applications. Acc. Chem. Res. 47, 550-559 (2014).

23. Wang, S. et al. Aptasensor with expanded nucleotide using DNA nanotetrahedra for electrochemical detection of cancerous exosomes. ACS Nano 11 3943-3949 (2017).

24. Lin, M. et al. Programmable engineering of a biosensing interface with tetrahedral DNA nanostructures for ultrasensitive DNA detection. Angew. Chem. 127, 2179-2183 (2015).

25. Wen, Y. et al. DNA nanostructure-based interfacial engineering for PCR-free ultrasensitive electrochemical analysis of microRNA. Sci. Rep. 2, 867-867 (2012).

26. Wen, Y. et al. DNA nanostructure-decorated surfaces for enhanced aptamertarget binding and electrochemical cocaine sensors. Anal. Chem. 83, 7418-7423 (2011)

27. Chen, $X$. et al. Ultrasensitive electrochemical detection of prostate-specific antigen by using antibodies anchored on a DNA nanostructural scaffold. Anal. Chem. 86, 7337-7342 (2014).

28. Goodman, R. P. et al. Rapid chiral assembly of rigid DNA building blocks for molecular nanofabrication. Science 310, 1661 LP-1661665 (2005).

29. Li, Z. et al. DNA Nanostructure-based universal microarray platform for highefficiency multiplex bioanalysis in biofluids. ACS Appl. Mater. Interfaces 6 17944-17953 (2014)

30. Pei, $H$. et al. Regenerable electrochemical immunological sensing at DNA nanostructure-decorated gold surfaces. Chem. Commun. 47, 6254-6256 (2011).

31. Zeng, D. et al. A novel ultrasensitive electrochemical DNA sensor based on double tetrahedral nanostructures. Biosens. Bioelectron. 71, 434-438 (2015).

32. DeOliveira, R. A. G., Materon, E. M., Melendez, M. E., Carvalho, A. L. \& Faria, R. C. Disposable microfluidic immunoarray device for sensitive breast cancer biomarker detection. ACS Appl. Mater. Interfaces 9, 27433-27440 (2017).

33. Hsieh, K., Patterson, A. S., Ferguson, B. S., Plaxco, K. W. \& Soh, H. T. Rapid, sensitive, and quantitative detection of pathogenic DNA at the point of care through microfluidic electrochemical quantitative loop-mediated isothermal amplification. Angew. Chem. Int. Ed. 51, 4896-4900 (2012).

34. Piraino, F., Volpetti, F., Watson, C. \& Maerkl, J. S. A digital-analog microfluidic platform for patient-centric multiplexed biomarker diagnostics of ultralow volume samples. ACS Nano 10, 1699-1710 (2016).

35. Singh, N. et al. Microporous nanocomposite enabled microfluidic biochip for cardiac biomarker detection. ACS Appl. Mater. Interfaces 9, 33576-33588 (2017). 
36. Soares, A. et al. Microfluidic-based genosensor to detect human papillomavirus (HPV16) for head and neck cancer. ACS Appl. Mater. Interfaces 10 36757-36763 (2018).

37. Uliana, C. V., Peverari, C. R., Afonso, A. S., Cominetti, M. R. \& Faria, R. C. Fully disposable microfluidic electrochemical device for detection of estrogen receptor alpha breast cancer biomarker. Biosens. Bioelectron. 99, 156-162 (2018).

38. Yan, M., Zang, D., Ge, S., Ge, L. \& Yu, J. A disposable electrochemical immunosensor based on carbon screen-printed electrodes for the detection of prostate specific antigen. Biosens. Bioelectron. 38, 355-361 (2012).

39. Yang, F. et al. A bubble-mediated intelligent microscale electrochemical device for single-step quantitative bioassays. Adv. Mater. 26, 4671-4676 (2014).

40. Li, Z. et al. Effect of surface pretreatment on self-assembly of thiol-modified DNA monolayers on gold electrode. J. Electroanalytical Chem. 722-723, 131-140 (2014).

41. Lillehoj, P. B., Wei, F. \& Ho, C.-M. A self-pumping lab-on-a-chip for rapid detection of botulinum toxin. Lab. Chip 10, 2265-2270 (2010).
42. Goodman, R. P., Berry, R. M. \& Turberfield, A. J. The single-step synthesis of a DNA tetrahedron. Chem. Commun. 12, 1372-1373 (2004).

43. Dupont, A. et al. Prostate specific antigen and prostatic acid phosphatase for monitoring therapy of carcinoma of the prostate. J. Urol. 146, 1064-1067 (1991).

44. Adel Ahmed, H. \& Azzazy, H. M. E. Power-free chip enzyme immunoassay for detection of prostate specific antigen (PSA) in serum. Biosens. Bioelectron. 49, 478-484 (2013).

45. Feng, Z., Zhi, S., Guo, L., Zhou, Y. \& Lei, C. An integrated magnetic microfluidic chip for rapid immunodetection of the prostate specific antigen using immunomagnetic beads. Mikrochimica Acta 186, 252-252 (2019).

46. Guo, X., Guo, Y., Liu, W., Chen, Y. \& Chu, W. Fabrication of paper-based microfluidic device by recycling foamed plastic and the application for multiplexed measurement of biomarkers. Spectrochimica Acta Part A: Mol. Biomol. Spectrosc. 223, 117341 (2019).

47. Chen, Y., Guo, X., Liu, W. \& Zhang, L. Paper-based fluorometric immunodevice with quantum-dot labeled antibodies for simultaneous detection of carcinoembryonic antigen and prostate specific antigen. Mikrochimica Acta 186, 112 (2019). 